\title{
Axisymmetric laminar flow of two-phase medium inside the system of two coaxial pipes
}

\section{Igor Kuzmenko,}

\section{Alexandre Gourjii}

National Technical University of Ukraine "Igor Sikorsky Kyiv Polytechnic Institute", Peremogy St. 37, 03056 Kyiv, Ukraine

Email:ozirno@ukr.net
The problem of the axisymmetric laminar flow of a two-phase (liquid-gas) flow inside the infinite system of coaxial tubes with a circular cross-section located vertically is considered. Analysis of the analytical solution showed that increase in velocity of the gas flow leads to the appearance of the hold-up regime (then the velocity of media at the interface is zero) and flooding regime (when volume flow of liquid is zero). It is discovered that these regimes occur at lower values of the velocity of the gas flow for the heated medium in comparison with the cold medium.

Keywords: coaxial tubes, two-phase flow, laminar flow, holdup regime, flooding regime

\footnotetext{
ABBREVIATIONS

$d p / d z, \mathrm{~Pa} / \mathrm{m}$ - pressure gradient in the pipe,

$U_{1}(r), \mathrm{m} / \mathrm{s}$ - velocity profile of gas,

$g, \mathrm{~m} / \mathrm{s}^{2}$ - acceleration of gravity,

$h, \mathrm{~m}$ - thickness of the water layer,

$Q_{1}, \mathrm{~m}^{3} / \mathrm{s}$ - volumetric flow of gas,

$Q_{2}, \mathrm{~m}^{3} / \mathrm{s}$ - volumetric flow of liquid in the layer placed near the internal surface of the channel, $Q_{3}, \mathrm{~m}^{3} / \mathrm{s}$ - volumetric flow of liquid in the layer placed near the external surface of the channel, $r, \mathrm{~m}$ - radial coordinate,

$R_{0}, \mathrm{~m} / \mathrm{s}$ - characteristic channel radius,

$R_{1}, \mathrm{~m}$ - internal channel radius,

$R_{2}, \mathrm{~m}$ - external channel radius,

$\operatorname{Re}=\left(U_{0} R_{0}\right) / v_{1}-$ Reynolds number,

$\mathrm{Re}_{\mathrm{cr} 1}$ - critical Reynolds number in the hold-up regime,

$\mathrm{Re}_{\mathrm{cr} 2}$ - critical Reynolds number in the flooding regime,

$U_{0}, \mathrm{~m} / \mathrm{s}$ - maximum gas velocity in the middle part of the channel,

$U_{2}(r), \mathrm{m} / \mathrm{s}$ - velocity profile of the liquid layer placed near the internal surface of the channel,

$U_{3}(r), \mathrm{m} / \mathrm{s}$ - velocity profile of the liquid layer placed near the external surface of the channel, $\bar{U}_{1}, \mathrm{~m} / \mathrm{s}$ - average velocity of gas,

$\bar{U}_{2}, \mathrm{~m} / \mathrm{s}$ - average velocity of liquid in the layer placed near the internal surface of the channel, $\bar{U}_{3}, \mathrm{~m} / \mathrm{s}$ - average velocity of liquid in the layer placed near the external surface of the channel, $z$, $\mathrm{m}$ - longitudinal coordinate,

$\mu_{1}$, Pa.s - dynamical viscosity coefficient of gas, $\mu_{2}$, Pa.s - dynamical viscosity coefficient of liquid,

$v_{1}, \mathrm{~m}^{2} / \mathrm{s}$ - kinematical viscosity coefficient of gas,

$\rho_{1}, \mathrm{~kg} / \mathrm{m}^{3}$ - density of gas,

$\rho_{2}, \mathrm{~kg} / \mathrm{m}^{3}-$ density of liquid.
} 


\section{INTRODUCTION}

Theoretical description of hydrodynamics in a contact apparatus without a separating wall, for example, cooling towers or contact economizers, presents a complex scientific problem. A definition of the flooding regime on the regular packing of a contact apparatus that indicates the transition to the bubble counterflow regime of liquid film and gas is important and relevant during operation of such devices. There are many theories to determine the flooding regime on the regular packing of a contact apparatus in scientific literature. These theories describe the hydrodynamics of the interface of the interacting phases at the regular packing of a contact apparatus, taking into account unsteady forces on this surface. In particular, many investigations apply the theory of stability.

The stability theory of travelling film, which can describe the interface evolution between two parallel flows, is often used for the qualitative analysis of physical regularities on the interface between two media. The restoring force (capillary force) can restore the broken interface to the original form. However, the surface does not recover immediately, inertial effects lead to the appearance of surface waves $[1,2]$. The amplitude of the waves can grow rapidly under certain conditions, the surface becomes unstable. As a result, the channel can go into a flooding regime [8].

Many researchers [4] apply the statistical equilibrium approach based on the equilibrium analysis between gravity force, shear stress and aerodynamic pressure on the interface. If the average fluid velocity in the thin liquid film tends to zero for channels oriented vertically, then the hold-up regime appears. It means that liquid does not move down.

This approach exists in some variations, but all of them require parameters of the wave motion, which can be determined experimentally.

Today the theory of soliton [5] is actively used in this problem. Water soliton can be observed on the interface at the flooding process even for moderate gas velocity in the channel. However, these models of soliton formation are developed mainly for flow-through horizontal flow and can be extended to an inclined counter-current flow.
The theory of separated motion [6] is based on the assumption that the flooding regime is the limit case for operating conditions when liquid or gas velocities have increased. In this case, stratified flows of liquid and gas are considered separate cylinders with the friction force describing their interaction. There are many models in modern scientific literature because of different boundary conditions and different approaches of the friction force.

Many researchers use a separate-flow model for analyzing physical processes within twophase channels [7]. They consider the water-gas interface based on a static equilibrium between the gravity force tangential stress and the pressure distribution that controls gas flow. If the average fluid velocity is zero, then it is assumed that the film has a hold-up regime, downward motion is absent. A separate cylinders model of the interaction of two-phase flows exists in various variations, but all of them require experimental determination of the parameters of motion $[8,9]$.

Recently, the theory of separated motion has fast developed $[10,11]$, which is based on the assumption that the flooding process occurs at the moment when the velocity of liquid or gas increases sharply. Stratified fluid and gas flows are considered in individual cylinders, the frictional force between which describes their interaction. Such a model has a number of varieties because of different boundary conditions and values of the coefficient of friction.

However, the use of the friction coefficient reduces the use of such models. In particular, in [7] a model for a friction in a laminar and turbulent motion is described separately. In addition, in [12] the flooding regime is described, if the friction between water-gas flows is neglected and the channel diameter is greater than $0.43 \mathrm{~m}$ at normal conditions.

In many cases, the dynamic range of the steady motion of the gas flow in contact devices is determined from the results of experimental studies for fixed pipe geometric dimensions [9]. These studies made it possible to reveal the main regularities and general trends in the interaction of two-phase flows in vertical segments of contact devices, the conditions for the appearance and development of various regimes of motion 
and interaction of liquid and gas in the interior space of contact devices for various purposes. Nevertheless, the quantitative data in these cases are of empirical nature for the specific geometric parameters of a contact apparatus and values of the volume flow of liquid and gas [10].

Also, one of the main tasks is the influence of the temperature regimes on the flooding regime at the laminar flow motion. Consideration of such influence in determining the regime of flooding allows to more accurately calculate the operating mode of a contact apparatus without a separating wall.

The main goal of this study is to construct a model of the regime of flooding without the value of the coefficient of friction on the water filmair boundary within the theory of separated motion. Using the constructed model, quantitative dependences of the volume flow rate of liquid on the flow rate of gas inside the vertical system of coaxial pipes (that correspond to the regular packing in cooling towers or contact economizers) were obtained. The results are obtained in the approximation of an axisymmetric, laminar flow of the water film-air at the flooding regime and close to them.

\section{MATHEMATICAL MODEL OF THE FLOW}

Let us consider a laminar stationary axisymmetric motion of a two-phase (gas-liquid) flow inside two coaxial infinite tubes with a circular cross-section (Fig. 1) located vertically. Let $R_{1}$ be the radius of the inner tube and $R_{2}$ the radius of the outer tube. A gas with a density $\rho_{1}$ and a dynamical viscosity coefficient $\mu_{1}$ fills the middle part of the annular space $\left(R_{1}+h \leq r \leq R_{2}-h\right)$, and a liquid with a density $\rho_{2}\left(\rho_{1}<<\rho_{2}\right)$ and dynamical viscosity coefficient $\mu_{2}$ adjoins hard surfaces $\left(R_{1} \leq r \leq R_{1}+h, R_{2}-h \leq r \leq R_{2}\right)$, where $h$ is the thickness of the liquid layer. A pressure gradient $d p / d z=$ const is formed in the pipe, which drives the gas in the upward direction. It is necessary to determine the distribution of the longitudinal velocity components of the media $U_{1}(r), U_{2}(r)$ and $U_{3}(r)$ in the cross-section of the tubes.

The governing equations describing the motion of media are the Navier-Stokes equations [11], which for an axisymmetric case in a cylin-

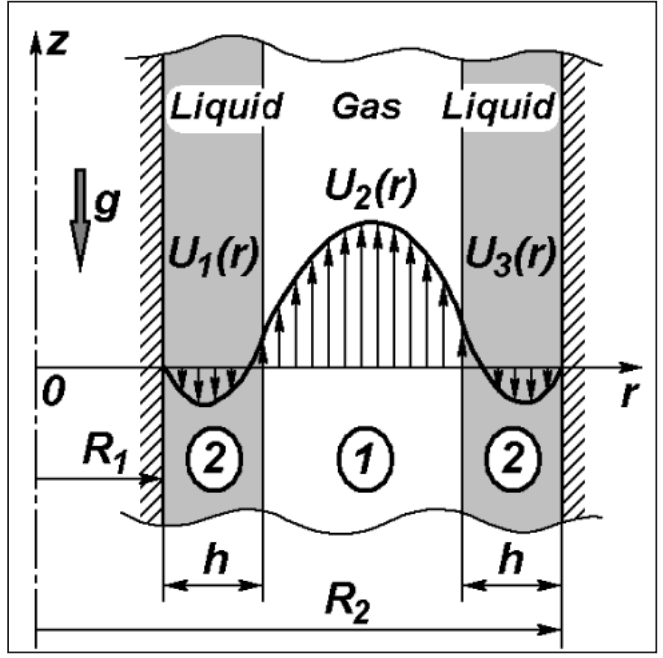

Fig. 1. Geometry of the problem

drical coordinate system $(r, z)$ reduce to a system of ordinary differential equations:

$$
\begin{aligned}
& \frac{\mu_{1}}{r} \frac{d}{d r}\left(r \frac{d U_{1}}{d r}\right)=\frac{d p}{d z} \text { for } \quad R_{1}+h \leq r \leq R_{2}-h, \\
& \frac{\mu_{2}}{r} \frac{d}{d r}\left(r \frac{d U_{2}}{d r}\right)=\rho_{2} g+\frac{d p}{d z} \text { for } R_{1} \leq r \leq R_{1}+h, \\
& \frac{\mu_{2}}{r} \frac{d}{d r}\left(r \frac{d U_{3}}{d r}\right)=\rho_{2} g+\frac{d p}{d z} \text { for } R_{2} \leq r \leq R_{2}+h,
\end{aligned}
$$

where $g$ is acceleration of gravity.

The mathematical formulation of the problem must be supplemented by the following boundary conditions:

$$
\begin{aligned}
& U_{2}\left(R_{1}\right)=0, U_{2}\left(R_{1}+h\right)=U_{1}\left(R_{1}+h\right), \\
& \left.\mu_{1} \frac{d U_{1}}{d r}\right|_{r=R_{1}+h}=\left.\mu_{2} \frac{d U_{2}}{d r}\right|_{r=R_{1}+h} \\
& U_{1}\left(R_{2}-h\right)=U_{3}\left(R_{2}-h\right), \\
& \left.\mu_{1} \frac{d U_{1}}{d r}\right|_{r=R_{2}-h}=\left.\mu_{2} \frac{d U_{3}}{d r}\right|_{r=R_{2}-h}, U_{3}\left(R_{2}\right)=0
\end{aligned} .
$$

The first and last conditions (2) are no-slip conditions for a viscous liquid to solid walls, the second and fourth equations express conjugation conditions for media with respect to velocity, and the third and fifth equations are conjugation conditions of media with respect to shear stress.

To perform a comparative analysis of flow parameters within coaxial tubes, it is necessary to dimension the equations of motion (1) and 
boundary conditions (2). We apply for normalizing the following values: $R_{0}=R_{2}, U_{0}, \rho_{1}$ and $\mu_{1}$. In this case, the dimensionless quantities (marked with an asterisk) have the following values:

$$
\begin{aligned}
& r^{*}=\frac{r}{R_{0}}, z^{*}=\frac{z}{R_{0}}, R_{1}^{*}=\frac{R_{1}}{R_{0}}, R_{2}^{*}=\frac{R_{2}}{R_{0}}=1.0, \\
& h^{*}=\frac{h}{R_{0}}, U_{1}^{*}=\frac{U_{1}}{U_{0}}, U_{2}^{*}=\frac{U_{2}}{U_{0}}, U_{3}^{*}=\frac{U_{3}}{U_{0}}, \\
& \rho^{*}=\frac{\rho_{2}}{\rho_{1}}, p^{*}=\frac{p}{\rho_{1} U_{0}^{2}}, \mu^{*}=\frac{\mu_{1}}{\mu_{1}}, g^{*}=\frac{g R_{2}}{U_{0}^{2}}, \\
& Q_{1}^{*}=\frac{Q_{1}}{U_{0} R_{0}^{2}}, Q_{2}^{*}=\frac{Q_{2}}{U_{0} R_{0}^{2}}, Q_{3}^{*}=\frac{Q_{3}}{U_{0} R_{0}^{2}} .
\end{aligned}
$$

Here, $Q_{1}, Q_{2}$ and $Q_{3}$ are the volumetric flow of media with a corresponding index. Here and below, the asterisks in dimensionless quantities are omitted.

Substitution of eqs. (3) into differential eqs. (1) leads to a normalized system of equations:

$$
\begin{aligned}
& \frac{1}{\operatorname{Re}} \frac{1}{r} \frac{d}{d r}\left(r \frac{d U_{1}}{d r}\right)=\frac{d p}{d z} \text { for } \quad R_{1}+h \leq r \leq R_{2}-h, \\
& \frac{1}{\operatorname{Re}} \frac{\mu}{r} \frac{d}{d r}\left(r \frac{d U_{2}}{d r}\right)=\rho g+\frac{d p}{d z} \text { for } R_{1} \leq r \leq R_{1}+h, \\
& \frac{1}{\operatorname{Re}} \frac{\mu}{r} \frac{d}{d r}\left(r \frac{d U_{3}}{d r}\right)=\rho g+\frac{d p}{d z} \text { for } R_{2}-h \leq r \leq R_{2} .
\end{aligned}
$$

The normalized boundary conditions take the following form:

$$
\begin{aligned}
& U_{2}\left(R_{1}\right)=0, U_{3}\left(R_{1}+h\right)=U_{1}\left(R_{1}+h\right), \\
& \left.\frac{d U_{1}}{d r}\right|_{r=R_{1}+h}=\left.\mu \frac{d U_{2}}{d r}\right|_{r=R_{1}+h}, \\
& U_{1}\left(R_{2}-h\right)=U_{3}\left(R_{2}-h\right), \\
& \left.\frac{d U_{1}}{d r}\right|_{r=R_{2}-h}=\left.\mu \frac{d U_{3}}{d r}\right|_{r=R_{2}-h}, U_{3}\left(R_{2}\right)=0 .
\end{aligned}
$$

Note that the normalized value of gravity acceleration can be expressed in terms of the Reynolds number in the notation defined before:

$$
g^{*}=\frac{g R_{0}}{U_{0}^{2}}=\frac{G}{\mathrm{Re}}, G=\frac{g R_{0}^{3} \rho_{1}^{2}}{\mu_{1}^{2}} .
$$

The first integration of eqs. (4) with respect to the variable $r$ leads to the following system of equations:

$$
\begin{aligned}
& r \frac{d U_{1}}{d r}=\frac{\operatorname{Re}}{2} \frac{d p}{d z} r^{2}+A \text { for } R_{1 H} \leq r \leq R_{2 H}, \\
& r \frac{d U_{2}}{d r}=\frac{\operatorname{Re}}{2 \mu}\left(\rho g+\frac{d p}{d z}\right) r^{2}+B \text { for } R_{1} \leq r \leq R_{1 H}, \\
& r \frac{d U_{3}}{d r}=\frac{\operatorname{Re}}{2 \mu}\left(\rho g+\frac{d p}{d z}\right) r^{2}+C \text { for } R_{2 H} \leq r \leq R_{2},
\end{aligned}
$$

where $A, B$ and $C$ are integration constants. Eqs. (4) use the notation: $R_{1 H}=R_{1}+h, R_{2 H}=R_{2}-h$.

The second integration of eq. (7) with respect to the variable $r$ allows us to recover unknown functions:

$$
\begin{aligned}
& U_{1}(r)=\frac{\operatorname{Re}}{4} \frac{d p}{d z} r^{2}+A \ln r+D \text { for } R_{1 H} \leq r \leq R_{2 H}, \\
& U_{2}(r)=\frac{\operatorname{Re}}{4 \mu}\left(\rho g+\frac{d p}{d z}\right) r^{2}+B \ln r+E \text { for } R_{1} \leq r \leq R_{1 H}, \\
& U_{3}(r)=\frac{\operatorname{Re}}{4 \mu}\left(\rho g+\frac{d p}{d z}\right) r^{2}+C \ln r+F \text { for } R_{2 H} \leq r \leq R_{2},
\end{aligned}
$$

where $D, E$ and $F$ are integration constants. All integration constants have to be determined from the boundary conditions (5).

The first boundary condition (5) for the second eq. (8) allows to express the integration constant $E$ in terms of the constant $B$. We obtain

$$
E=\frac{\operatorname{Re}}{4 \mu}\left(\rho g+\frac{d p}{d z}\right) R_{1}^{2}-B \ln R_{1}
$$

By analogy, the last boundary condition (5) in the third eq. (8) gives equation for the constant $F$ in terms of the constant $C$. In this case,

$$
E=\frac{\operatorname{Re}}{4 \mu}\left(\rho g+\frac{d p}{d z}\right) R_{1}^{2}-B \ln R_{1}
$$

Therefore, the solution of the problem (8) can be represented now in the following form:

$$
\begin{aligned}
& U_{1}(r)=\frac{\operatorname{Re}}{4} \frac{d p}{d z} r^{2}+A \ln r+D \text { for } R_{1 H} \leq r \leq R_{2 H}, \\
& U_{2}(r)=\frac{\operatorname{Re}}{4 \mu}\left(\rho g+\frac{d p}{d z}\right)\left(r^{2}-R_{1}^{2}\right)+B \ln \frac{r}{R_{1}} \\
& \text { for } R_{1} \leq r \leq R_{1 H}, \\
& U_{3}(r)=\frac{\operatorname{Re}}{4 \mu}\left(\rho g+\frac{d p}{d z}\right)\left(r^{2}-R_{2}^{2}\right)+C \ln \frac{r}{R_{2}} \\
& \text { for } R_{2 H} \leq r \leq R_{2},
\end{aligned}
$$

The substitution of the third boundary condition (5) into the system of eqs. (11) leads to the equality 


$$
A=\frac{\operatorname{Re}}{2} \rho g R_{1 H}+B \mu
$$

Similarly, substituting the fifth boundary condition (5) into the system of eqs. (11) allows us to express the integration constant $C$ through the constant $B$. We obtain

$$
C=\frac{\operatorname{Re}}{2 \mu} \rho g\left(R_{1 H}^{2}-R_{2 H}^{2}\right)+B .
$$

At this stage of the construction of the solution, the system of eqs. (11) already contains only two constants of integration:

$$
\begin{aligned}
& U_{1}(r)=\frac{\operatorname{Re}}{4} \frac{d p}{d z} r^{2}+\frac{\operatorname{Re}}{2} \rho g R_{1 H}^{2} \ln r+B \mu \ln r+D \\
& \text { for } R_{1 H} \leq r \leq R_{2 H}, \\
& U_{2}(r)=\frac{\operatorname{Re}}{4 \mu}\left(\rho g+\frac{d p}{d z}\right)\left(r^{2}-R_{1}^{2}\right)+B \ln \frac{r}{R_{1}} \\
& \text { for } R_{1} \leq r \leq R_{1 H}, \\
& U_{3}(r)=\frac{\operatorname{Re}}{4 \mu}\left(\rho g+\frac{d p}{d z}\right)\left(r^{2}-R_{2}^{2}\right)+\frac{\operatorname{Re}}{2 \mu} \rho g R_{12}+B \ln \frac{r}{R_{2}} \\
& \text { for } R_{2 H} \leq r \leq R_{2},
\end{aligned}
$$

with notation $R_{12}=\left(R_{1}+h\right)^{2}-\left(R_{2}-h\right)^{2}$.

The second boundary condition (5) allows us to express the integration constant $D$ in terms of the value of the constant $B$. Substitution of eq. (14) into this boundary condition gives the following equality:

$$
\begin{aligned}
& D=\frac{\operatorname{Re}}{4 \mu}\left(\rho g+\frac{d p}{d z}\right)\left[R_{1 H}^{22}-R_{1}^{2}\right]+ \\
& +B\left[\ln \frac{R_{1 H}}{R_{1}}-\mu \ln R_{1 H}\right]-\frac{\operatorname{Re}}{4} R_{1 H}^{2}\left\{\frac{d p}{d z}+2 \rho g \ln R_{1 H}\right\} .
\end{aligned}
$$

Finally, the fourth boundary condition (5) allows us to determine the value of the integration constant:

$$
\begin{aligned}
& B\left[\ln \frac{R_{2 H}}{R_{2}}-\ln \frac{R_{1 H}}{R_{1}}-\mu \ln \frac{R_{2 H}}{R_{1 H}}\right]= \\
= & \frac{\operatorname{Re}}{2} \rho g R_{1 H}^{2} \ln \frac{R_{2 H}}{R_{1 H}}-\frac{\operatorname{Re}}{4} \frac{d p}{d z} R_{12}+ \\
+ & \frac{\operatorname{Re}}{4 \mu}\left[R_{12}-R_{1}^{2}+R_{2}^{2}\right]-\frac{\operatorname{Re}}{2 \mu} \rho g R_{12} \ln \frac{R_{2 H}}{R_{2}} .
\end{aligned}
$$

Thus, the solution of problem (4) with boundary conditions (5) can be represented in the following form:

$$
\begin{aligned}
& U_{1}(r)=\frac{\operatorname{Re}}{4} \frac{d p}{d z}\left[r^{2}-R_{1 H}^{2}\right]+\frac{\operatorname{Re}}{2} \rho g R_{1 H}^{2} \ln \frac{r}{R_{1 h}}+ \\
& +\frac{\operatorname{Re}}{4 \mu}\left(\rho g+\frac{d p}{d z}\right)\left[R_{1 H}^{2}-R_{1}^{2}\right]+B\left[\mu \ln \frac{r}{R_{1 H}}+\ln \frac{R_{1 H}}{R_{1}}\right] \\
& \text { for } R_{1} \leq r \leq R_{1 H}, \\
& U_{2}(r)=\frac{\operatorname{Re}}{4 \mu}\left(\rho g+\frac{d p}{d z}\right)\left(r^{2}-R_{1}^{2}\right)+B \ln \frac{r}{R_{1}} \\
& \text { for } \quad R_{1} \leq r \leq R_{1 H}, \\
& U_{3}(r)=+\frac{\operatorname{Re}}{4 \mu}\left(\rho g+\frac{d p}{d z}\right)\left(r^{2}-R_{2}^{2}\right)+\frac{\operatorname{Re}}{2 \mu} \rho g R_{12} \ln \frac{r}{R_{2}}+B \ln \frac{r}{R_{2}} \\
& \text { for } R_{2 H} \leq r \leq R_{2},
\end{aligned}
$$

taking into account eq. (16) and the notations adopted earlier.

To determine volumetric flow of media, it is necessary to calculate the integrals

$$
\begin{aligned}
& Q_{1}=2 \pi \int_{R_{1 R_{2}}}^{R_{2 H}} U_{1}(r) r d r, \quad Q_{2}=2 \pi \int_{R_{1}}^{R_{1 H}} U_{2}(r) r d r \\
& Q_{3}=2 \pi \int_{R_{2 H}}^{U_{2}} U_{3}(r) r d r .
\end{aligned}
$$

The substitution of the solution (17) into the integrals (18) and the subsequent integration within these limits leads to the following expressions:

$$
\begin{aligned}
& Q_{1}=\frac{\operatorname{Re} \pi}{8}\left\{\frac{d p}{d z}\left(R_{2 H}^{4}-R_{2 H}^{4}\right)+\right. \\
& +\left(8 \rho g R_{1 H}^{2}+\frac{16 \mu}{\operatorname{Re}} B\right)\left[R_{2 H}^{2}\left(\frac{\ln R_{2 H}}{2}-\frac{1}{4}\right)-\right. \\
& \left.-R_{1 H}^{2}\left(\frac{\ln R_{1 H}}{2}-\frac{1}{4}\right)\right]+\left[\frac{2}{\mu}\left(\rho g+\frac{d p}{d z} B\right)\left(R_{1 H}^{2}-R_{1}^{2}\right)+\right. \\
& +\frac{8 B}{\operatorname{Re}} \ln \frac{R_{1 H}}{R_{1}}-2 \frac{d p}{d z} R_{1 H}^{2}-4 \rho g R_{1 H}^{2} \ln R_{1 H^{-}} \\
& \left.\left.-\frac{8 B \mu}{\operatorname{Re}} \ln R_{1 H}\right]\left(R_{2 H}^{2}-R_{1 H}^{2}\right)\right\} \text {, } \\
& Q_{2}=\frac{\operatorname{Re} \pi}{8}\left\{\frac{1}{\mu}\left(\rho g+\frac{d p}{d z}\right)\left(R_{1 H}^{4}-R_{1}^{4}\right)+\right. \\
& +\frac{16 B}{\operatorname{Re}}\left[R_{1 H}^{2}\left(\frac{\ln R_{1 H}}{2}-\frac{1}{4}\right)-\right. \\
& \left.-R_{1}^{2}\left(\frac{\ln R_{1}}{2}-\frac{1}{4}\right)\right]-\left[\frac{2}{\mu}\left(\rho g+\frac{d p}{d z}\right) R_{1}^{2}+\right. \\
& \left.\left.+\frac{8 B}{\mu} \ln R_{1}\right]\left(R_{1 H}^{2}-R_{1}^{2}\right)\right\} \text {, }
\end{aligned}
$$




$$
\begin{aligned}
& Q_{3}=\frac{\operatorname{Re} \pi}{8}\left\{\frac{1}{\mu}\left(\rho g+\frac{d p}{d z}\right)\left(R_{2}^{4}-R_{2 H}^{4}\right)+\right. \\
& +\left(\frac{8 \rho g}{\mu}\left(R_{1 H}^{2}-R_{2 H}^{2}\right)+\frac{16 \mathrm{~B}}{\operatorname{Re}}\right)\left[R_{2}^{2}\left(\frac{\ln R_{2}}{2}-\frac{1}{4}\right)-\right. \\
& \left.-R_{2 H}^{2}\left(\frac{\ln R_{2} H}{2}-\frac{1}{4}\right)\right]-\left[\frac{2}{\mu}\left(\rho g+\frac{d p}{d z}\right) R_{2}^{2}+\right. \\
& \left.\left.+\left(\frac{4 \rho g}{\mu}\left(R_{1 H}^{2}-R_{2 H}^{2}+\frac{8 B}{\operatorname{Re}}\right) \ln R_{2}\right)\right]\left(R_{2}^{2}-R_{2 H}^{2}\right)\right\} .
\end{aligned}
$$

To determine the mean velocity values over the cross-section, one can use the following equations:

$$
\bar{U}_{1}=\frac{Q_{1}}{\pi R_{1 H}^{2}}, \bar{U}_{2}=\frac{Q_{2}}{\pi\left(R_{2 H}^{2}-R_{1 H}^{2}\right)}, \bar{U}_{3}=\frac{Q_{3}}{\pi\left(R_{2}^{2}-R_{2}^{2}\right)}
$$

These equations will be used for performing a numerical analysis of physical parameters of a two-phase flow inside two coaxial tubes.

\section{NUMERICAL ANALYSIS}

Let us consider the features of a stationary twophase flow (air-water) inside an infinite channel formed by two coaxial tubes. The air flow moves in a vertical direction; the liquid is located near the hard surfaces. To carry out the dimensionless quantitative analysis, let us consider the case with the following characteristic parameters: $R_{0}=R_{2}=0.005 \mathrm{~m}, \rho_{1}=\mu p_{0} / R T$ is the gas density for isothermal flow in the temperature range $0 \ldots 100^{\circ} \mathrm{C}$ (excluding limit values at atmospheric pressure), where $R=8.34 \mathrm{~J} /(\mathrm{mol} \cdot \mathrm{deg})$ is the universal gas constant, $\mu=0.029 \mathrm{~kg} / \mathrm{mol}$ is molar air weight, $p_{0}=10^{5} \mathrm{~Pa}$ is an atmospheric pressure.

The dynamical viscosity coefficient of air and water in this range of temperatures varies within fairly wide limits $[12,13]$. The filled circles in the figure show the tabulated values. It is shown that the viscosity of the gas $\mu_{1}$ increases in a first approximation linearly with respect to the temperature $T$, and the water viscosity $\mu_{2}$ decreases with increasing temperature. That is why the ratio of the dynamical viscosity coefficients $\mu=\mu_{1} / \mu_{2}$ varies widely. Consequently, it can be assumed that the quantitative dependences of the gas and liquid flows rates should differ in the temperature range under consideration. Therefore, when carrying out further analysis, we select three characteristic cases: regime $A\left(T=1^{\circ} \mathrm{C}\right), \mu=$ 103.5; regime $B\left(\mathrm{~T}=50^{\circ} \mathrm{C}\right), \mu=28.0$; and regime $C\left(T=99^{\circ} \mathrm{C}\right), \mu=13.0$.

The negative pressure gradient $d p / d z=$ const, applied to the coaxial channel system, drives the gas motion in the middle part of the section in the direction from the bottom to the top. It is known [13] that the parabolic velocity profile of the gas is formed inside the channel for small Reynolds numbers. At the media interface, the equality of shear viscous stresses entrains a part of the liquid on the surface in a vertical motion, forming on the surface a flow with a velocity different from zero. The direction motion of the liquid at the media interface is determined by the ratio of the shear viscous stress force upward and the gravity of the liquid directed downward. Examples of gas and liquid velocity profiles within coaxial tubes are shown in Fig. 2 for different values of Reynolds numbers for the case $\mu=28.0$ (regime B), $h=0.02 . R_{1}=0.8$. Here, $U(r)=U_{2}(r)$ for $R_{1} \leq r \leq R_{1 H}, U(r)=U_{1}(r)$ for $R_{1 H} \leq r \leq R_{2 H}$, and $U(r)=U_{3}(r)$ for $R_{1 H} \leq r \leq R_{2}$. The features of the velocity distribution near the inner surface of the channel are shown in the figure with the index " $b$ ".

The dependencies presented in Fig. 2 were obtained as a result of the solution of the transcendental equation, in which the right-hand side of the first equation (17) is zero for $r=R_{1}+h$. Following the second boundary condition (5), analogous results can be obtained by equating the second equation (17) to zero for $r=R_{1}+h$. Studies show that the parameters of the hold-up regime for the inner layer of the liquid practically coincide with the parameters of the hold-up regime for the outer layer of the liquid. Note that in the second case it is necessary to equate the second or third eq. (17) to zero for $r=R_{2}-h$. The difference in these parameters for the inner and outer layers depends on the values of the inner radius $R_{1}$. An increase in the value of $R_{1}$ leads to a decrease in the difference in the values of the corresponding parameters. In the range of values $R_{1}=0.1 \ldots 0.9$, this difference does not exceed $\approx 0.1 \%$ and does not actually appear in the graphs presented in this research.

At low gas velocities, the gravity force for liquid is predominant, and the flow velocity of the liquid takes on negative values at all radii values 
(a)
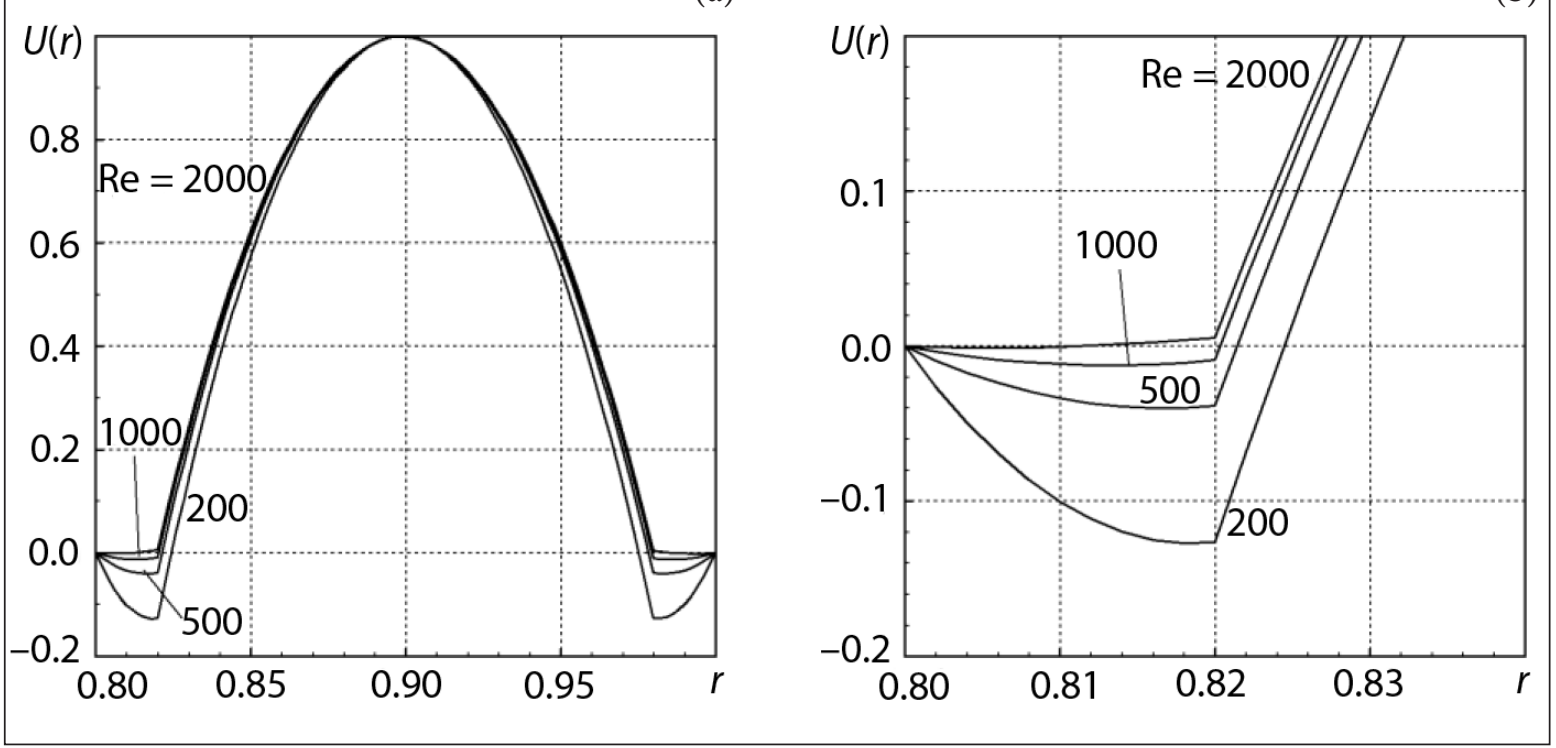

Fig. 2. The flow velocity profile in the cross-section of the channel (a) and near the inner surface of the channel (b) for $h=0.02, R_{1}=0.8$

in the cross-section of the channel. An increase in the velocity of the gas leads to an increase in the gradient of the component of the axial velocity of the gas and, as a result, to an increase in the shear viscous stress on the media interface. The velocity of the liquid downward increases by module (see the case $\mathrm{Re}=200$ in Fig. 2); however, in the whole range of radial values the liquid velocity assumes negative values. In this case, the maximum value of the velocity of the liquid is in the layer adjacent to the media interface.

At $\operatorname{Re}=1000$, the liquid velocity profile changes. The maximum value of the liquid velocity is shifted to the inner region of the liquid layer. At the interface between the media, the liquid velocity asymptotically tends to zero. Subsequently, the hydrodynamic regime, in which the value of the velocity of the liquid (or gas) on the media interface has a zero value, is conditionally called the hold-up regime. Note that in this case the volumetric flow rate of the liquid takes on negative values, because the liquid flows down the entire section of the liquid layer.

A further increase in the rate of gas velocity leads to the case which is characterized by a change in the sign of the velocity of the near-surface liquid layer. Under the action of viscous shear stress, the liquid can move upward (for example, the case $\operatorname{Re}=2000$ ). At the same time, part of the liquid adjacent to the interface of the media moves upward, while part of the fluid adjacent to the solid surface moves downward under the action of gravity. Therefore, a case can appear in which positive and negative fluid flows through the cross-section of the channel compensate each other. Subsequently, the hydrodynamic regimes, in which the volumetric flow rate of the liquid in the channel cross-section achieves a zero value is conditionally called the flooding regime.

The value of the gas velocity at which the hold-up regime arises depends not only on the ratio of the dynamical viscosity coefficients of the fluid and gas, but also on the geometry of the channel and the thickness of the liquid layer. Figure 3 illustrates the dependence of critical values of Reynolds number $\mathrm{Re}_{\mathrm{cr} 1}$ (or corresponded gas velocity) in the hold-up regime on the thickness $h$ of the liquid layer for certain values of the radii $R_{1}$ of the inner tube and the temperature of the media. It is shown that an increase in the thickness of the liquid layer first leads to an increase in the gas velocity at which the hold-up regime arises. For large values of $h$, the corresponding Reynolds numbers decrease. The critical value of Reynolds numbers $\mathrm{Re}_{\mathrm{cr} 1}$ for large values of $\mu$ (cold media) becomes larger in comparison with the cases in which $\mu$ takes smaller values (hot media). 


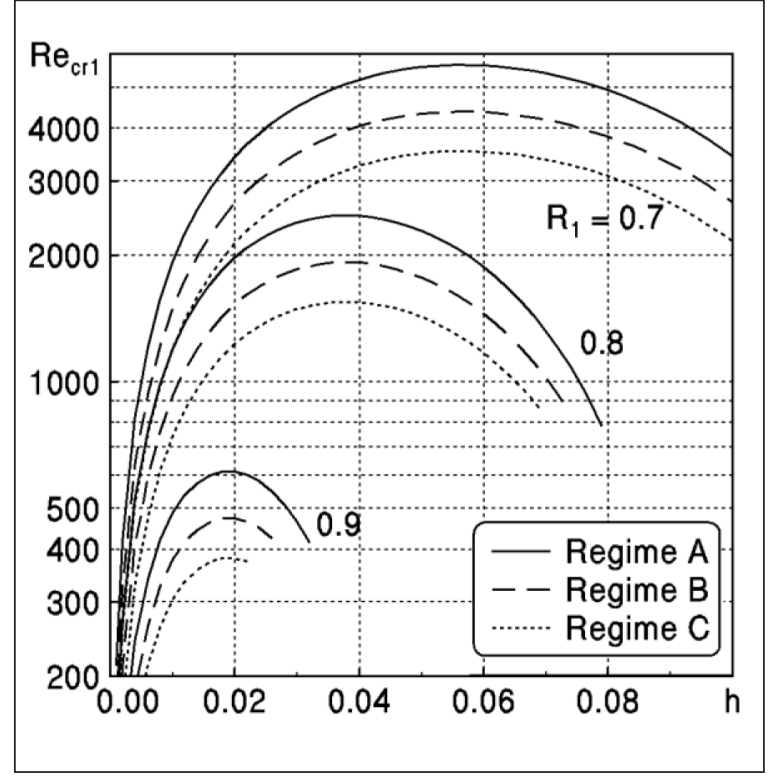

Fig. 3. Dependence of the critical Reynolds number $\mathrm{Re}_{\mathrm{c} 1}$ in the hold-up regime on the thickness $h$ of the liquid layer for different values of the radii $R_{1}$ of the inner tube

It is interesting to note that the critical values of the Reynolds numbers $\mathrm{Re}_{\mathrm{cr} 1}$ for the holdup regime decrease gradually for large values of the thickness $h$ of the liquid layer. This effect is explained by the mutual influence of the liquid layer on the inner surface and the liquid layer on the outer surface. As the values of $h$ increase, the thickness of the gas layer in the cross-section of the channel decreases. In this case, the gradient of the longitudinal component of the gas velocity near the interface increases. This leads to an increase in the viscous shear stress. For this reason, the hold-up regime occurs at lower Reynolds numbers Re.

Analysis of scientific literature $[1,7]$ shows that the gas flow in a narrow channel $\left(R_{2}-R_{1}-2 \mathrm{~h}\right)$ acquires a dynamic instability, which is accompanied by a violation of the symmetry of the flow, the formation of a wave surface at the media interface. In some cases, this can lead to the rupture of the surface of the liquid, the formation of droplets of different diameters. Note that the surface tension forces play an important role in this process. Similar effects can be observed with an increase in the gas velocity to values characteristic of the transition and turbulent gas flow regimes. Some details of the physical effects that arise in the turbulent flow of a two-phase medium inside an infinite tube can be found in the monograph [1]. For this reason, the increasing theoretical branches of the dependencies in Fig. 3 can be considered physically unstable solutions; their observation in real currents is problematic.

The dependence of the critical values of the Reynolds numbers $\mathrm{Re}_{\mathrm{crl}}$ under which the flooding regime on the radius $R_{1}$ of the inner tube at fixed values of the thickness of the liquid layer is shown in Fig. 4. There is a general tendency associated with a decrease in the velocity of the gas flow with increasing radius $R_{1}$. Note that the critical value of Re decreases with decreasing value of $\mu$. In other words, the process of hold up in a heated system occurs at lower values of the gas velocity inside the pipe system under consideration.

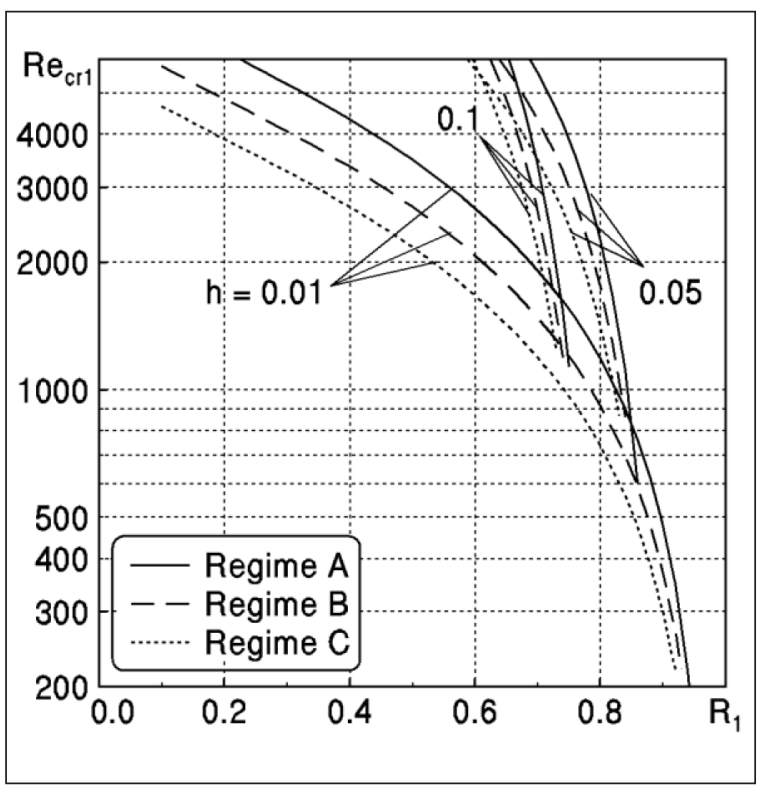

Fig. 4. Dependence of Reynolds numbers $\mathrm{Re}_{\mathrm{cr} 1}$ in the hold-up regime on the radii $R_{1}$ of the inner tube for different values $h$ of the liquid layer

Figure 5 illustrates the dependence of the volumetric flow of the gas and the liquid on the gas velocity inside the coaxial tubes for the various regimes mentioned earlier. The figure shows the case: $R_{1}=0.8, h=0.02$. It can be seen that this dependence is nonlinear. An increase in the values of Reynolds numbers leads to an increase in the values of the dimensionless volumetric flow of the gas (3). At $\operatorname{Re} \approx 1000 \ldots 2000$, the curves gradually reach a constant value. Hence, starting with these 
Reynolds numbers Re, the volumetric flow of the gas becomes directly proportional to the gas velocity inside the coaxial tubes. Note that increase in the temperature of a two-phase medium leads to a displacement of this effect in the region of large values of the gas velocity.

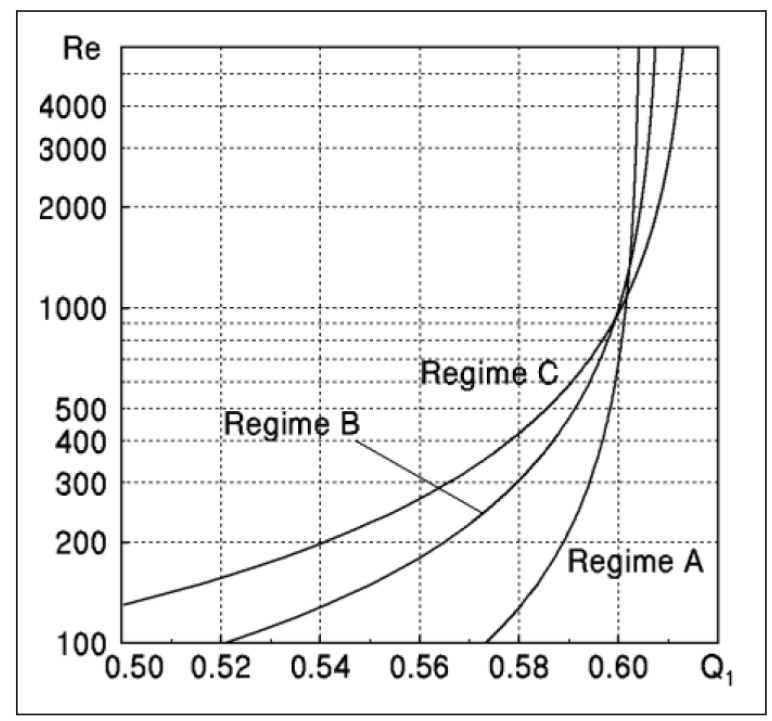

Fig. 5. Dependence of Reynolds numbers Re for regimes $A, B$ and $C$ on volumetric flow of gas $Q_{1}$

The dependence of values of Reynold numbers on the normalized volumetric flow of liquids for different regimes is shown in Fig. 6 $\left(R_{1}=0.8, h=0.02\right)$. The dashed line in the figure shows the $\operatorname{Re}\left(Q_{2}\right)$ dependence, and the dotted line shows the $\operatorname{Re}\left(Q_{3}\right)$ dependence. The solid line shows the total volumetric flow $Q_{\mathrm{F}}=Q_{2}+Q_{3}$. It is shown that increase in the gas velocity leads to a change in the direction of liquid motion. When $Q=0$, a flooding regime of liquid occurs inside the coaxial tube system. We note that the flooding regime for a cold two-phase medium occurs at large values of the gas velocities in comparison with the analogous case for a heated medium.

Figure 7 illustrates the dependence of the critical Reynolds number $\mathrm{Re}_{\mathrm{cr} 2}$ values, at which the flooding regime occurs, on the thickness of the liquid layer for different values of the radius $R_{1}$ of the inner tube. Increasing the thickness of the liquid layer requires large values of the gas velocity. However, at some value of $h$, the opposite solid surface begins to influence, and the

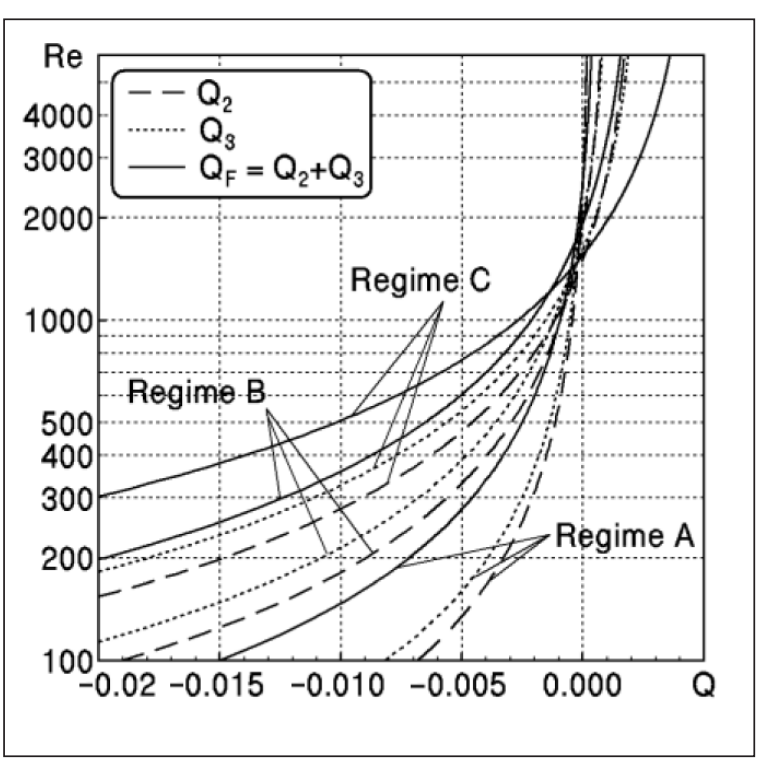

Fig. 6. Dependence of Reynolds numbers Re for regimes $A, B$ and $C$ on volumetric liquid flows $Q_{1}, Q_{2}$ and $Q_{F}$

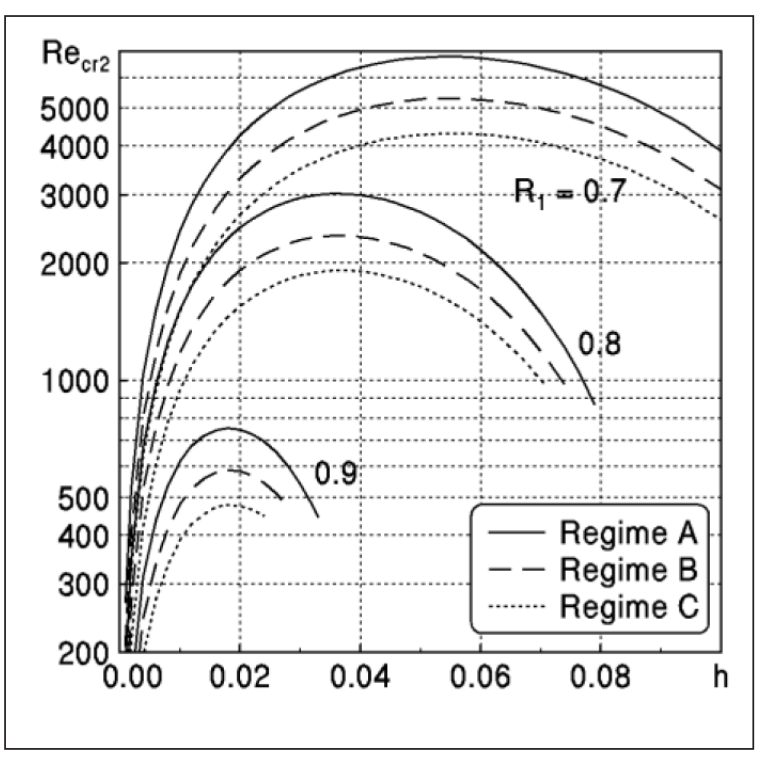

Fig. 7. Dependence of the critical Reynolds number $\operatorname{Re}_{\mathrm{cr} 2}$ for the flooding regime on the thickness $h$ of the liquid layer for different values of the radii $R_{1}$ of the inner tube

values of the gas velocity gradually begin to decrease. This trend takes place for different temperatures of the two-phase mixture. Nevertheless, the flooding regime occurs at lower critical Reynolds numbers over the entire range of values of $h$ for a heated two-phase medium.

The dependence of the critical Reynolds number $\mathrm{Re}_{\mathrm{cr} 2}$ values, at which the flooding regime occurs, of the normalized radius $R_{1}$ of 
the inner tube for different thicknesses $h$ of the liquid layer is shown in Fig. 8. It is shown that the flooding regime occurs at lower gas velocities for large values of $R_{1}$. Simultaneously, the general tendency is preserved: the flooding regime for a heated two-phase medium occurs at lower Reynolds numbers Re.

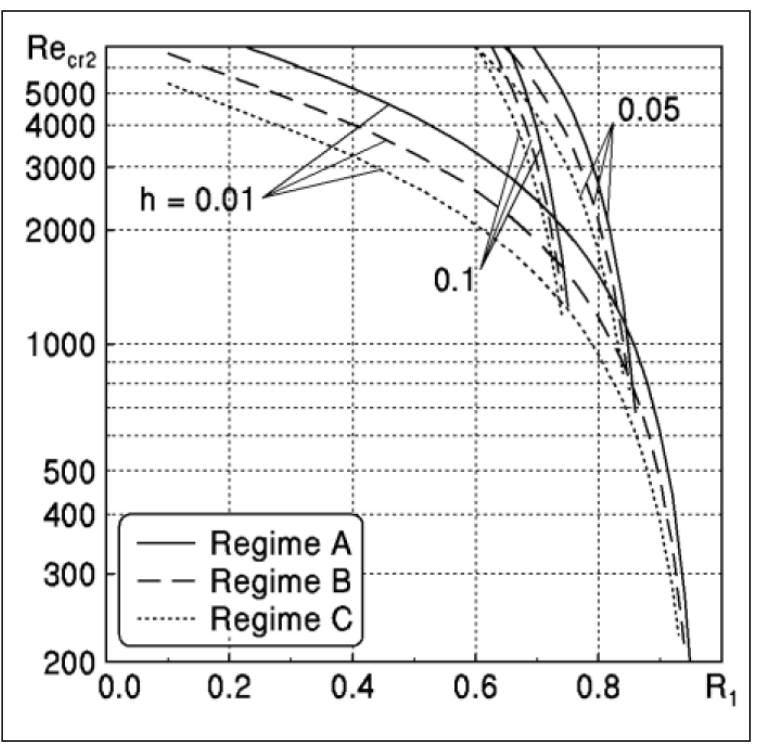

Fig. 8. Dependence of the Reynolds number $\mathrm{Re}_{\mathrm{c} 2}$ for the flooding regime on the inner tube radii $R_{1}$ for different values of $h$ of the liquid layer

\section{CONCLUSIONS}

The problem of the interaction of a two-phase flow (liquid-gas) inside a system of vertical coaxial tubes with a circular cross-section is considered. The basic equations describing the motion of a viscous, axisymmetric flow are $\mathrm{Na}$ vier-Stokes equations, which for a laminar flow regime reduce to a system of ordinary differential equations. This mathematical problem has an analytical solution.

Analysis of the numerical solution of the problem shows that the gravity force of the liquid is the predominant force at low gas velocities, and liquid velocity takes negative values for all radii in the cross-section of the channel. An increase in the velocity of the gas leads to an increase in the gradient of the axial component of the gas velocity and, as a result, an increase in viscous shear stress at the media interface.
At the interface between media, this force entrains a part of the liquid on the surface in a vertical motion, forming a flow on the surface with non-zero velocity. The direction of the liquid motion at the media interface is determined by the ratio of the viscous shear stress force directed upward and the gravity force of the liquid directed downward.

For certain values of the gas velocity, a holdup regime may occur, which is characterized by vanishing of the velocity of the liquid (or the gas) at the media interface. Increase in the thickness of the liquid layer leads to increase in the gas velocity, at which the hold-up regime occurs. Further increasing the values of $h$, the corresponding values of the gas velocity decrease. In this case, the gradient of the longitudinal component of the gas velocity near the interface increases as shown on Fig. 3 for $h>0.04$ at $R_{1}=0.7$. This leads to increase in the viscous shear stress force and, as a result, the hold-up regime occurs at lower Reynolds numbers Re. If radius $R_{1}$ of the inner tube increases and the dimensionless value of $h$ is a constant, then gas velocity in the channel for the hold-up regime decreases. This regime occurs in the system at lower values of gas velocity inside the channel under consideration.

The flooding regime, which follows the holdup regime, has similar properties. This regime is determined by the condition of equality to zero of the volumetric flow of the liquid in the cross-section of the channel.

Received 17 March 2018 Accepted 15 May 2018

\section{References}

1. Bezrodny M. K., Pioro I. L., Kostyuk T. O. Transfer Processes in Two-Phase Thermosyphon Systems. Theory and Practice. Kyiv: Fact, 2005. 704 p.

2. Levich V. G. Physical-Chemical Hydrodynamics. M: Fizmatgiz, 1959. 700 p.

3. Vijayan M., Jayanti S., Balakrishnan A. R. Experimental study of air-water countercurrent annular flow under post-flooding conditions. International Journal of Multiphase Flow. 2002. Vol. 28. P. 51-67. 
4. Kutateladze S. S., Styrikovich M. A. Hydrodynamics of Gas-Fiquid Systems M: Energia, 1976. $296 \mathrm{p}$.

5. Bankoff S. G., Lee S. C. A Critical Review of the Flooding Literature. Evanston: Northwestern University, 1983. $121 \mathrm{p}$.

6. Labuntzov D. A., Yagov V. V. Mechanics of TwoPhase Systems. M: MEI, 2000. 374 p.

7. Klyuev N., Solov'eva E. Mathematical Models of Two-Phase Flows: Textbook. Samara: Samara University, 2010. $51 \mathrm{p}$.

8. Park I., Mudawar I. Climbing film, flooding and failing film behavior in upflow condensation. International Journal of Heat and Mass Transfer. 2013. Vol. 65. P. 44-61.

9. Nada S. A. Cooling of very hot vertical tubes by falling liquid film in presence of countercurrent flow of rising gases. International Journal of Thermal Sciences. 2015. Vol. 88. P. 228-237.

10. Berrichona J. D., Louahlia-Gualous H., Bandelier Ph., Clement P., Bariteau N. Experimental study of flooding phenomenon in a power plant reflux air-cooled condenser. Applied Thermal Engineering. 2015. Vol. 79. P. 214-224.

11. Celata G. P., Cumo M., Serato T. A data set of flooding in circular tube. Experimental Thermal and Fluid Science. 1992. Vol. 5. P. 437-447.

12. Vijayan M., Jayanti S., Balakrishnan A. R. Effect of tube diameter on flooding. International Journal of Multiphase Flow. 2001. Vol. 27. P. 797-816.
13. Lamb H. Hydrodynamics (6th ed.). Cambridge: Cambridge University Press, 1932. 928 p.

14. Kikoin I. K. Tables of Physical Quantities. M: Atomizdat, 1976. 1008 p.

15. Kaye G. W. C., Laby T. H. Tables of Physical and Chemical Constants. London: Longmans Green \& Co, 1986. 432 p.

Igor Kuzmenko, Alexandre Gourjii

AKSISIMETRINIS LAMINARINIS TEKE்JIMAS

DVIFAZINIAME SRAUTE DVIEJŲ

KOAKSIALINIŲ VAMZDŽIŲ SISTEMOJE

\section{Santrauka}

Aptariama aksisimetrinio laminarinio tekejjimo dviejų fazių (skystis-dujos) srauto begalinèje koaksialių apvalaus skerspjūvio vertikalių vamzdžių sistemoje problema. Analitinio sprendinio analizè parodé, kad dujų srauto greičio padidèjimas veda prie stabdymo režimo (tada terpès srauto greitis sąsajoje yra lygus nuliui) ir užliejimo režimo (kai skysčio srautas yra lygus nuliui). Nustatyta, kad šie režimai pasireiškia esant mažesniems dujų srauto greičiams šildomoje terpejje, palyginti su šalta terpe.

Raktažodžiai: koaksialiniai vamzdžiai, dvifazis srautas, laminarinis tekejjimas, pasipriešinimo režimas, užliejimo režimas 